\title{
Uso de los servicios de salud por la población de 60 años y más en México
}

\author{
S. Aída Borges-Yáñez, C.D., M.S.P., ${ }^{(1)}$ Héctor Gómez-Dantés, M.C., M. en C. ${ }^{(2)}$
}

\section{Borges-Yáñez SA, Gómez-Dantés H. Uso de los servicios de salud por la población de 60 años y más en México. Salud Publica Mex I 998;40: I 3-23.}

\section{Resumen}

Objetivo. Describir las características sociodemográficas y las determinantes de la utilización de los servicios de salud por la población de 60 años y más en México. Material y métodos. De la información captada en la Encuesta Nacional de Salud II se obtuvieron datos de 4628 personas ancianas; se analizaron las prevalencias de las principales enfermedades crónicas y en los individuos que utilizaron los servicios de salud dos semanas antes de la encuesta se investigaron los factores determinantes de su utilización. Resultados. Las enfermedades crónicas más frecuentemente notificadas fueron: hipertensión arterial, diabetes mellitus y cardiopatías. La tasa de utilización de servicios en ese grupo de edad fue de II.4\%, y de hospitalización 5.5\%. Los servicios de salud más utilizados fueron: el médico particular, el Instituto Mexicano del Seguro Social (IMSS), la Secretaría de Salud, el Instituto de Seguridad y Servicios Sociales de los Trabajadores del Estado y el IMSS-Solidaridad. De 25 a $45 \%$ de los derechohabientes no hacen uso de estos servicios $y$, en su lugar, acuden a los servicios privados. Las variables explicativas más importantes de la utilización de servicios fueron: el estar enfermo, no haber trabajado la semana anterior y ser derechohabiente. El género, la zona de residencia y el grado de escolaridad no fueron significativos en la explicación de la utilización de servicios de salud. Conclusiones. El estudio demuestra la necesidad de fortalecer los programas preventivos y de apoyo para esta población, ya que sólo acude a los servicios de salud cuando está enferma.

Palabras clave: servicios de salud/utilización; anciano; seguridad social; hipertensión; diabetes mellitus no insulinodependiente; cardiopatías; México

\author{
Borges-Yáñez SA, Gómez-Dantés H. \\ Health service utilization by the 60 -year-old \\ and older population in Mexico. \\ Salud Publica Mex 1 998;40:I 3-23.
}

\begin{abstract}
Objective. To describe the sociodemographic characteristics and determinants of health services utilization by the Mexican population of 60 years of age or more. Materials and methods. Information obtained from the National Health Survey II (ENSA-II) allowed analysis of 4628 elderly people. Prevalence of chronic diseases was analyzed and the determinants of individuals who had used the health services in the two weeks prior to the interview. Results. The chronic diseases most frequently reported were: hypertension, diabetes and heart disease. The utilization and hospitalization rates of that age group were 11.4 and $5.5 \%$, respectively. The services most frequently used were: the private physician, social security (IMSS, ISSSTE) welfare services (Ministry of Health -SSA- and IMSS-Solidaridad). Interestingly, 25 to $45 \%$ of social security insurance holders did not use the services, instead they consulted a private physician. The most important explanatory variables for health services utilization were: the perceived illness, not having worked for the previous week and being a social security insurance holder. Gender, area of residence and level of education were not significant in the explanation of health services utilization. Conclusions. This study demonstrates the need to strengthen preventive and support programs for the elderly population, since they only demand health services when they feel sick.
\end{abstract}

Key words: health services/utilization; aged; social security; diabetes mellitus, non-insulin-dependent; heart diseases; Mexico

(I) Dirección General de Epidemiología, Secretaría de Salud, México.

(2) Centro de Investigación sobre Enfermedades Infecciosas, Instituto Nacional de Salud Pública, México. 
$\mathrm{E}$ n el pasado era sumamente difícil llegar a la vejez; esa posibilidad se consideraba como un privilegio y hablaba de la fortaleza y del vigor de los que la lograban. La vejez se consideraba como una etapa de la vida rica en experiencia y sabiduría, por lo que se respetaba a los ancianos. En México, la atención del anciano, elemento básico en la cohesión familiar, era un aspecto fundamental de la dinámica familiar, así como de las redes sociales de apoyo, lo cual garantizaba una mejor calidad de vida para los viejos. En la sociedad moderna -individualista y competitiva- se valora más la juventud y la condición física, lo que, de manera indirecta, debilita las redes sociales de apoyo a los ancianos que, poco a poco, van perdiendo su lugar protagónico en la familia. El viejo se ha convertido en una carga familiar, social y económica, después de haber dedicado los mejores años de su vida más productiva en construir la sociedad que ahora lo rechaza.

La evolución de las instituciones prestadoras de los servicios de salud es un ejemplo muy concreto de la respuesta social hacia la vejez, ya que ilustra cómo la sociedad invirtió en la salud de la población económicamente activa (PEA) -con servicios de salud diseñados para atender a los trabajadores y sus familias- sin anticipar las demandas de atención que resultarían de la transición demográfica producto del aumento en la esperanza de vida, el descenso de la mortalidad infantil, materna y general, el mejoramiento de la nutrición ${ }^{1}$ y la disminución de la fecundidad.

Por otro lado, el mejoramiento de las condiciones generales de vida y el mayor acceso a los servicios de salud han provocado un descenso en las tasas de mortalidad por enfermedades infecciosas y un ascenso marcado de las enfermedades crónicas -tumores malignos, diabetes mellitus y enfermedades cardiovasculares- como las principales causas de muerte. Estas son las que, de manera natural, afectan más a los ancianos. ${ }^{2}$ El incremento actual y futuro de la población anciana obliga a replantear la organización de los servicios, a fin de poder satisfacer la futura demanda.

Para el año 2020,12\% de la población total mundial tendrá más de 60 años y, debido a la migración del campo a la ciudad, se estima que para el año 2000, $75 \%$ de los ancianos residirán en áreas urbanas., ${ }^{3,4}$ En México los sujetos de 60 años y más representan $6.5 \%$ del total de la población, ${ }^{5}$ cifra muy similar a la de otros países de América Latina, como Argentina (9.6\%), Chile (6.7\%) y Brasil (5.2\%). ${ }^{6}$ Sin embargo, en Cuba (12.2\%) y Uruguay (12.2\%) esa proporción es semejante a la de países desarrollados como la ex Unión Soviética $(10 \%)$, Alemania (10\%), Estados Unidos de América -EUA$(12.3 \%),{ }^{7}$ Canadá $(12 \%)$ y Europa $(13 \%) .{ }^{8-10}$
En 1960, en México, la esperanza de vida era de 57 años para los hombres y 60 años para las mujeres, $y$ en 1992 se incrementó a 67 y 73.7 años, respectivamente. ${ }^{11,12}$ Las diferencias en la esperanza de vida por género indican que los hombres adultos tienen factores de riesgo que los hacen proclives a enfermedades crónicas, con un desenlace fatal a edades más tempranas. ${ }^{4,13,14}$ Por el contrario, las mujeres adultas tienden a los padecimientos crónicos discapacitantes que afectan su calidad de vida durante mayor tiempo. Lo anterior influye directamente sobre las diferencias en los motivos de consulta y hospitalización, y sobre la mayor utilización de los servicios de salud que hacen las mujeres. ${ }^{9,15,16}$

La naturaleza de los problemas de salud de la población anciana plantea retos importantes para el Sector Salud: la mayor frecuencia de padecimientos crónicos, ${ }^{17}$ su diagnóstico a edades más tempranas y la producción de distintos tipo de discapacidades, darán lugar a una demanda de servicios más grande y compleja. La atención institucional enfrentará, a corto plazo, por lo menos dos tipos de demandas: en el primer nivel de atención será necesario prevenir los riesgos a edades más tempranas, destacando los aspectos positivos de la salud para mejorar la calidad de vida y disminuyendo la discapacidad física y psicológica derivada de la demencia, la depresión, el abandono y la pérdida de las redes sociales de apoyo. ${ }^{18}$ Por otro lado, el incremento en la prevalencia de las enfermedades crónicas se combinará para generar una demanda de servicios altamente especializados, de alto costo, que requerirán de atención multidisciplinaria. ${ }^{19,20}$

Un hecho indiscutible es que la población anciana es una de las mayores usuarias de los servicios de salud. En 1983, en México, se notificó que 79\% de los ancianos residentes en zonas urbanas y $67 \%$ de los que habitaban las zonas suburbanas habían utilizado servicios médicos en los doce meses previos al estudio. ${ }^{21} \mathrm{~A}$ pesar de las escasas evidencias y de las estimaciones, se desconocen los datos referentes a las características sociodemográficas y la magnitud de la utilización que hace actualmente ese segmento de la población de los servicios médicos. En consideración a lo anterior, la Encuesta Nacional de Salud II (ENSA-II) brinda una excelente oportunidad para analizar la utilización de los servicios de salud de la población anciana en el ámbito nacional. El objetivo de este trabajo es presentar el perfil sociodemográfico de la población de 60 años y más en México y la utilización de servicios de salud, por género, zona de residencia e institución de atención, con base en los datos recolectados en la ENSA-II. El análisis de la información será de utilidad en la 
planeación de los servicios preventivos y curativos, y en la búsqueda de alternativas de atención de mayor calidad y menor costo, con el propósito de disminuir las discapacidades y mejorar la calidad de vida de los ancianos durante mayor tiempo.

\section{Material y métodos}

Como parte de la estrategia para conocer el estado de salud de la población, desde 1986 la Secretaría de Salud (SSA) ha llevado a cabo diversas encuestas nacionales. En 1994 se realizó la ENSA-II cuyos objetivos fueron identificar la cobertura de los servicios de salud, los perfiles de utilización, los niveles de accesibilidad, la calidad de los servicios y el monto de los gastos en salud de acuerdo con la percepción de los usuarios. Se realizó una selección de viviendas a través de una encuesta probabilística de diseño polietápico, estratificado y por conglomerados. Se dividió al país en cinco regiones y se aseguró un mínimo muestral de 100 viviendas en los estados más pequeños, utilizando el marco muestral jurisdiccional de la Dirección General de Epidemiología (DGE). La metodología y el marco muestral de la ENSA-II se presentan detalladamente en otros documentos. ${ }^{22}$

El universo de estudio consistió en 12615 viviendas, y a partir de las entrevistas con los responsables de los hogares se obtuvo información de 61524 personas de todas edades. El análisis que se presenta corresponde a 4628 individuos de 60 años y más, es decir, $7.5 \%$ del total de la muestra. En este estudio se analiza el perfil sociodemográfico, la percepción del estado de salud individual, la morbilidad crónica, el consumo de medicamentos y remedios caseros, y el tipo de servicios de salud utilizados durante los 12 meses previos a la encuesta.

En el análisis se identificaron 527 ancianos que utilizaron los servicios de salud. La utilización de servicios se definió como "la asistencia/demanda a un servicio de salud curativo, preventivo o tradicional que hizo la población los 15 días previos al levantamiento de la información". Se obtuvo información específica a través de una entrevista individual, indagando el motivo de la demanda de atención, la duración de la enfermedad, el tipo de institución a la que acudieron, las causas para no utilizar el mismo servicio, la percepción de la calidad de la atención recibida y del costo de los servicios utilizados.

Se obtuvieron frecuencias de las variables mencionadas y se analizó su relación por género, zona de residencia e institución de atención. Además, se determinaron las tasas de utilización de servicios médicos por género, estado civil (casado, unión libre/separado, divorciado/soltero, viudo), zona de residencia (rural/ urbana), condición de derechohabiencia (sí/no), ocupación (activo/inactivo), escolaridad (primaria completa y posprimaria/primaria incompleta), edad, condición de enfermedad (sí/no), trabajó la semana previa (sí/no) y analfabetismo (sí/no). Para analizar la asociación entre las variables sociodemográficas y la utilización de servicios se utilizó un modelo de regresión logística. Los datos se encuentran en una base de datos en DBase IV, y el análisis se realizó con el paquete estadístico STATA 3.1 (Stata Corporation).

\section{Resultados}

La ENSA-II contiene información de 4628 individuos de 60 años y más: $48.4 \%$ son hombres (edad promedio $=70$ años), y $51.6 \%$, mujeres (edad promedio $=70.2$ años). La distribución por grupo de edad y sexo según zona de residencia urbana y rural no mostró diferencias, aunque la proporción de mujeres en la población se incrementa en los grupos de edad mayor (cuadro I).

El nivel de escolaridad en este grupo de edad es primordialmente la primaria, con diferencias por género, ya que los hombres tienen un mayor número de años de estudio que las mujeres. Por zona de residencia se encontró que sólo 1\% de los ancianos en las zonas rurales tienen un grado de escolaridad mayor que primaria (cuadro I). Es interesante señalar que aproximadamente $20 \%$ de las personas son analfabetas. Al observar la distribución de analfabetismo por género y zona de residencia, se encontró que $65 \%$ (853) son mujeres y, de éstas, $59 \%$ viven en las zonas rurales y $31 \%$ en ciudades.

Respecto al estado civil, gran parte de los hombres encuestados están casados o viven en unión libre (78\%) y $15 \%$ son viudos, a diferencia de las mujeres, entre las cuales la proporción de casadas es menor (46\%), y de viudas, mucho mayor (41\%). Por zona de residencia existe mayor proporción de ancianos casados o en unión libre $(66.8 \%)$ y menor de viudos $(26.2 \%)$ en las zonas rurales que en las zonas urbanas (cuadro I). Lo anterior señala la importancia que, respecto a la mortalidad, tienen los factores de riesgo propios de las grandes urbes.

En el rubro de ocupación, la población se clasificó en económicamente activa e inactiva, y esta última se subclasificó en: a) jubilados y pensionados; b) dedicados al hogar, y c) otros. La distribución por género indicó que $52.6 \%$ de los hombres y $10.3 \%$ de las mujeres son todavía económicamente activos. Gran parte de los ancianos inactivos son jubilados o pensionados, a diferencia de las mujeres, cuya mayoría se mantiene activa en las labores del hogar. El promedio de edad para 


\section{Cuadro I}

Características sociodemográficas de las personas de 60 años y más. México, 1994

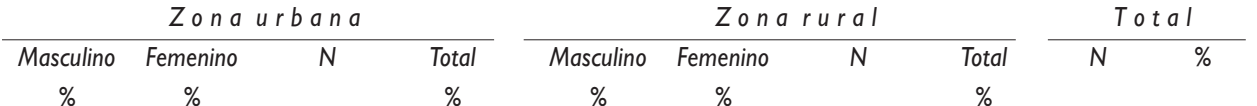

Edad

\begin{tabular}{|c|c|c|c|c|c|c|c|c|c|c|}
\hline $60-64$ & 33.4 & 32.9 & 1040 & 33.2 & 33.0 & 34.4 & 502 & 33.7 & 1542 & 33.3 \\
\hline $65-69$ & 21.8 & 22.4 & 695 & 22.2 & 22.4 & 22.0 & 331 & 22.2 & 1026 & 22.2 \\
\hline $70-74$ & 17.9 & 17.8 & 560 & 17.9 & 17.1 & 14.4 & 236 & 15.8 & 796 & 17.2 \\
\hline $75-79$ & 11.4 & 9.2 & 321 & 10.2 & 9.6 & 9.1 & 140 & 9.4 & 461 & 10.0 \\
\hline $80-89$ & 13.3 & 15.2 & 450 & 14.3 & 15.4 & 15.4 & 230 & 15.4 & 680 & 14.7 \\
\hline $90-99$ & 2.1 & 2.4 & 71 & 2.3 & 2.5 & 4.6 & 52 & 3.5 & 123 & 2.7 \\
\hline Total & 100.0 & 100.0 & 3137 & 100.0 & 100.0 & 100.0 & | 49| & 100.0 & 4628 & 100.0 \\
\hline
\end{tabular}

Escolaridad

\begin{tabular}{|c|c|c|c|c|c|c|c|c|c|c|}
\hline Primaria & 79.2 & 86.9 & 2537 & 83.4 & 99.1 & 98.8 & I 444 & 99.0 & 3981 & 88.4 \\
\hline Posprimaria & 20.8 & 13.1 & 506 & 16.6 & 0.9 & 1.2 & 15 & 1.0 & 521 & 11.6 \\
\hline Total & 100.0 & 100.0 & 3043 & 100.0 & 100.0 & 100.0 & I 459 & 100.0 & 4502 & 100.0 \\
\hline
\end{tabular}

Estado civil

\begin{tabular}{|c|c|c|c|c|c|c|c|c|c|c|}
\hline Unión libre, casado & 77.9 & 43.1 & 1852 & 59.2 & 78.7 & 53.4 & 994 & 66.8 & 2846 & 61.7 \\
\hline Divorciado, separado & 2.6 & 6.0 & 138 & 4.4 & 2.3 & 2.9 & 38 & 2.6 & 176 & 3.8 \\
\hline Viudo & 15.3 & 42.3 & 932 & 29.8 & 14.8 & 39.0 & 389 & 26.2 & $\mid 32$ | & 28.6 \\
\hline Soltero & 4.1 & 8.6 & 205 & 6.6 & 4.2 & 4.7 & 66 & 4.4 & 271 & 5.9 \\
\hline Total & 100.0 & 100.0 & $3 \mid 27$ & 100.0 & 100.0 & 100.0 & I 487 & 100.0 & 4614 & 100.0 \\
\hline
\end{tabular}

Ocupación

\begin{tabular}{|c|c|c|c|c|c|c|c|c|c|c|}
\hline Económicamente activo & 45.5 & 10.3 & 833 & 26.6 & 65.9 & 10.2 & 592 & 39.8 & I 425 & 30.9 \\
\hline Inactivo & 54.5 & 89.7 & 2298 & 73.4 & 34.1 & 89.8 & 896 & 60.2 & 3194 & 69.1 \\
\hline Total & 100.0 & 100.0 & $3|3|$ & 100.0 & 100.0 & 100.0 & 1488 & 100.0 & 4619 & 100.0 \\
\hline Jubilado, pensionado & 50.1 & 6.6 & 495 & 21.5 & 8.2 & 0.9 & 28 & 3.2 & 523 & 16.4 \\
\hline Hogar & 7.7 & 85.4 & 1350 & 58.8 & 7.8 & 89.2 & 580 & 64.7 & 1930 & 60.4 \\
\hline Otros & 42.2 & 8.0 & 453 & 19.7 & 84.0 & 9.9 & 288 & 32.1 & 741 & 23.2 \\
\hline Total inactivos & 100.0 & 100.0 & 2298 & 100.0 & 100.0 & 100.0 & 896 & 100.0 & 3194 & 100.0 \\
\hline
\end{tabular}

Derechohabiencia

\begin{tabular}{|c|c|c|c|c|c|c|c|c|c|c|}
\hline No & 40.1 & 43.3 & 1306 & 41.8 & 82.7 & 81.6 & 1217 & 82.2 & 2523 & 54.8 \\
\hline IMSS & 44.8 & 40.2 & 1321 & 42.3 & 12.3 & 12.7 & 185 & 12.5 & 1506 & 32.7 \\
\hline ISSSTE & 11.6 & 12.5 & 377 & 12.1 & 3.6 & 4.6 & 60 & 4.1 & 437 & 9.5 \\
\hline Otros & 3.5 & 4.0 & 117 & 3.8 & 1.4 & I.I & 19 & 1.2 & 136 & 3.0 \\
\hline Total & 100.0 & 100.0 & 3121 & 100.0 & 100.0 & 100.0 & $|48|$ & 100.0 & 4602 & 100.0 \\
\hline
\end{tabular}

Fuente: Secretaría de Salud; Encuesta Nacional de Salud II, México, 1994

los jubilados fue de 70.9 años; para los que se dedican al hogar, de 69.8, y para los activos, de 68.1 años. Por lugar de residencia, la proporción de ancianos inactivos es mayor en las zonas urbanas; existe un porcentaje mínimo de jubilados o pensionados (3.1\%) en la zona rural (cuadro I).
La condición de derechohabiencia mostró que no existen diferencias por género, ya que la proporción de derechohabientes es similar entre hombres $(46 \%)$ y mujeres $(45 \%)$. En cuanto a la cobertura, el Instituto Mexicano del Seguro Social (IMSS) proporciona servicios de salud a aproximadamente $30 \%$ y el Instituto 
de Seguridad y Servicios Sociales de los Trabajadores del Estado (ISSSTE) a 10\% de los ancianos. En contraste, al analizar esta condición por zona de residencia, $82 \%$ de los ancianos en la zona rural y $42 \%$ en la zona urbana carecen de servicios de seguridad social y pueden recurrir a la SSA y al IMSS-Solidaridad.

Entre las enfermedades crónicas informadas con mayor frecuencia en el grupo de 60 años y más destacan la hipertensión arterial (9.9\%); diabetes mellitus (9\%); cardiopatía (3.3\%) y ceguera, sordera o parálisis (2.8\%). Sólo se encontraron diferencias por género en la prevalencia de hipertensión arterial, siendo ésta mayor en las mujeres (12.9\%) que en los hombres $(6.6 \%)$ (figura 1). Por otra parte, se observa una mayor prevalencia de diabetes, hipertensión arterial, enfermedades del corazón y úlcera gástrica en los residentes de las zonas urbanas y mayor prevalencia de discapacidades y asma en las zonas rurales.

En cuanto a la percepción del estado de salud en el último año, $14 \%$ de los sujetos perciben su salud como mala o muy mala, independientemente del sexo, aunque en general los hombres tienden a notificar una mejor condición de salud. Los ancianos de las zonas urbanas indican tener mejor estado de salud (43\%) que aquellos de las zonas rurales (36\%). Es importante mencionar que la estimación de estas prevalencias se basa en el reporte hecho por la persona responsable del hogar, por lo que es probable que esas frecuencias estén subestimadas.

Durante el último año, 5.5\% de los hombres y $5.8 \%$ de las mujeres fueron hospitalizados; la principal causa

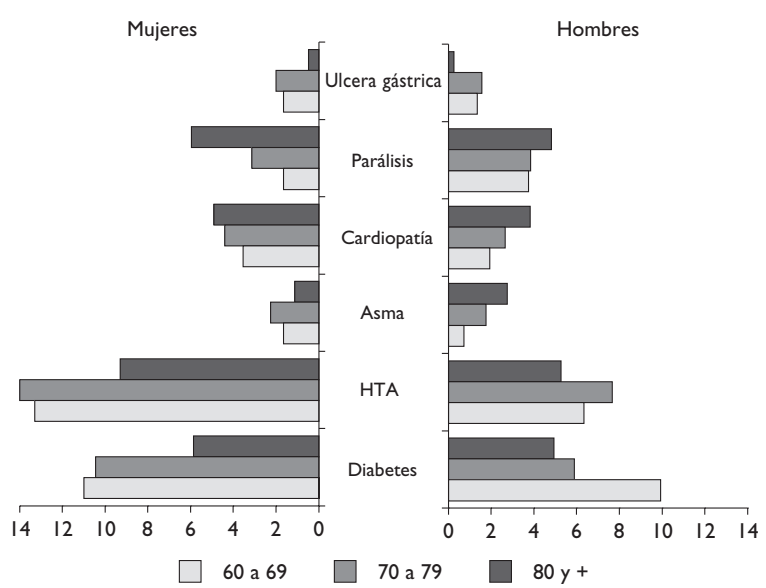

Fuente: Encuesta Nacional de Salud II, Secretaría de Salud, México, 1994

Figura I. Prevalencia de enfermedades crónicas NOTIFICADAS POR SEXO Y GRUPO DE EDAD, ENSA-II

salud pública de méxico / vol.40, no.1, enero-febrero de 1998 fue algún tipo de enfermedad (52.5\%), seguida por cirugía $(39.7 \%)$ y accidente $(7.9 \%)$. Las causas fueron significativamente diferentes, ya que un mayor porcentaje de hombres (10\%) que de mujeres $(5 \%)$ fueron hospitalizados por accidente. El IMSS absorbió el mayor porcentaje de hospitalizaciones (40.5\%). Por zona de residencia, $11.7 \%$ de las hospitalizaciones en zona rural se debieron a accidentes, a diferencia de $5.9 \%$ en la zona urbana.

El 20.5\% de los ancianos informó haber tenido algún problema de salud en las dos semanas previas al levantamiento de la encuesta. La edad promedio de los ancianos que utilizaron servicios de salud fue de 69.6 años. La tasa de utilización de los servicios de salud fue de $11.4 \%$, esto es, la tasa más alta de todos los grupos de edad (tasa nacional 5.8\%). En los mayores de 60 años, las tasas de utilización variaron entre 5 y $15 \%$, y las mujeres fueron las que más utilizaron los servicios en este periodo $(60 \%)$ y las que presentaron la tasa de utilización más alta. El $66.2 \%$ de los usuarios en las zonas rurales y $30.4 \%$ en las urbanas no tenían derecho a la seguridad social. Los servicios de salud donde más frecuentemente se atienden los ancianos en zonas urbanas son: el médico particular (38\%), el IMSS (32\%), la SSA (11.5\%), el ISSSTE (7.7\%) y el IMSS-Solidaridad $(2.5 \%)$. En zonas rurales son: el médico particular $(36 \%)$, la SSA (30\%), el IMSS-Solidaridad (13\%), el IMSS (10\%), y el ISSSTE (3\%). Cabe señalar que entre las personas jubiladas $2.3 \%$ no demandó atención médica, 26\% acudió al médico particular y $72 \%$ recibió atención en las instituciones a las que tienen derecho. El 21.4\% de las zonas rurales y $51.6 \%$ de las urbanas utilizó servicios como derechohabiente del IMSS; el resto acudió a otros servicios médicos.

En general, los ancianos derechohabientes solicitan atención médica en la institución correspondiente; sin embargo, una proporción considerable de esos asegurados también utiliza los servicios médicos privados. El 25\% de los derechohabientes del IMSS y $45 \%$ de los del ISSSTE acuden a otros servicios. Entre la población abierta, $60 \%$ recurrió al médico particular y $30 \%$ a la SSA (figura 2).

Entre las personas que indicaron haber tenido un problema de salud en las dos semanas previas, se indagó sobre el consumo de medicamentos y remedios caseros. El 74\% de los encuestados notificaron haber tomado medicamentos; lo anterior fue más frecuente entre las mujeres (75.9\%) que entre los hombres (70.5\%). En cambio, se encontró que $35.5 \%$ había consumido remedios caseros, más entre las mujeres (37.1\%) que entre los hombres (31\%). El consumo de medicamentos fue 1.7 (IC 95\% 1.25-2.29) veces mayor en las zonas urbanas que en las rurales, mientras que el de remedios 


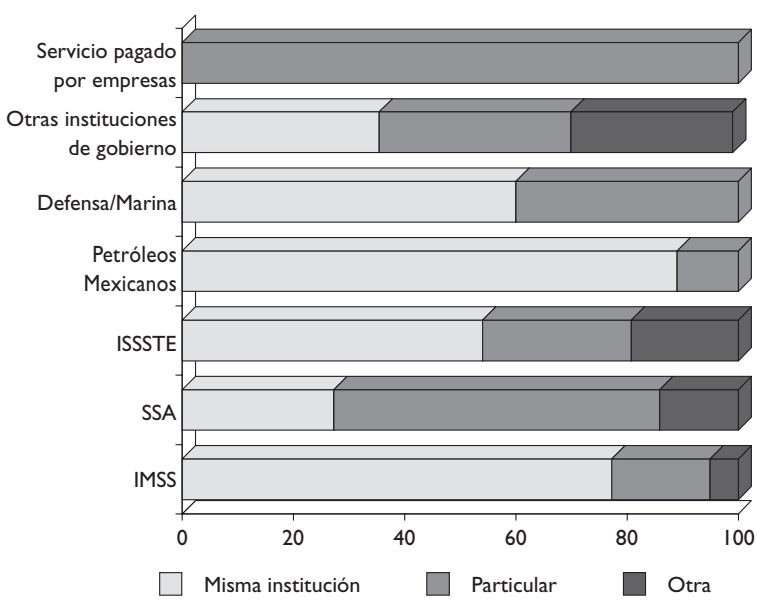

Fuente: Encuesta Nacional de Salud II, Secretaría de Salud, México, 1994

Figura 2. Porcentaje de derecho-habientes que UTILIZAN LOS SERVICIOS MÉDICOS A LOS QUE TIENEN DERECHO, ENSA-II

caseros fue superior en las zonas rurales que en las urbanas (razón de momios-RM-2.3, IC 95\% 1.71-3.02). Un hallazgo de importancia es que el consumo de medicamentos y remedios caseros es independiente de la severidad del problema de salud reportado.

En cuanto a la calidad de los servicios recibidos, $78 \%$ la consideró buena o muy buena; sin embargo, sólo $22 \%$ informó alguna mejoría a partir de la atención recibida. Respecto a la percepción del costo de los servicios, los usuarios consideraron que los particulares eran caros, y los otorgados por el IMSS, la SSA y el ISSSTE, económicos. De los usuarios, $90 \%$ opinó que regresarían al mismo lugar para ser atendidos y $9.3 \%$ no los volverían a utilizar. Las principales razones para no utilizar de nuevo los servicios de salud fueron: la mala atención, que el paciente no se curó y el alto costo del servicio.

El análisis inicial indicó las variables, examinadas una por una, que fueron estadísticamente significativas al establecer su asociación con la utilización de servicios (cuadro II). La tasa de utilización de servicios entre la población anciana fue mayor en las mujeres ( $R M=1.5)$, en la población analfabeta $(\mathrm{RM}=1.24)$, en los que no trabajaron $(\mathrm{RM}=2.1) \mathrm{y}$, sobre todo, en los que indicaron haber tenido algún problema de salud durante las dos semanas previas a la encuesta.

En el análisis de regresión logística la variable dependiente fue la utilización de los servicios en las

\section{Cuadro II}

TASAS DE UTILIZACIÓN DE SERVICIOS DE SALUd EN LAS DOS SEMANAS PREVIAS AL LEVANTAMIENTO DE LA encuesta, VARiables Seleccionadas. México, 1994

\begin{tabular}{|c|c|c|c|c|}
\hline Variable & Tasa & $R M$ & $p$ & IC $95 \%$ \\
\hline Enfermos & 40.1 & 16.15 & 0.00 & $13.08-19.90$ \\
\hline No enfermos & 3.9 & & & \\
\hline Femenino & 13.3 & 1.49 & 0.00 & I.24-I.79 \\
\hline Masculino & 9.3 & & & \\
\hline No sabe leer & 12.0 & 1.24 & 0.04 & $1.01-1.53$ \\
\hline Sí sabe leer & 9.9 & & & \\
\hline Primaria completa & 11.5 & .87 & 0.36 & $0.64-1.17$ \\
\hline Posprimaria & 10.2 & & & \\
\hline No trabajó semana pasada & 13.5 & 2.12 & 0.00 & $1.69-2.68$ \\
\hline Sí trabajó semana pasada & 6.8 & & & \\
\hline Zona urbana & 12.1 & 1.25 & 0.03 & $1.02-1.52$ \\
\hline Zona rural & 9.9 & & & \\
\hline Jubilado & 13.0 & 1.19 & 0.22 & $0.89-1.57$ \\
\hline Otros (activos e inactivos) & 11.2 & & & \\
\hline Casado/ unión libre & 10.9 & I & - & - \\
\hline Viudo/ soltero & 11.9 & 1.11 & 0.28 & $0.91-1.35$ \\
\hline Separado/divorciado & 14.8 & 1.42 & 0.10 & $0.90-2.23$ \\
\hline Sí derechohabiencia & 15.0 & 1.92 & 0.00 & $1.59-2.30$ \\
\hline No derechohabiencia & 8.4 & & & \\
\hline
\end{tabular}

Fuente: Encuesta Nacional de Salud II, Secretaría de Salud, México, 1994

últimas dos semanas. En el modelo completo se confirmaron las variables significativas encontradas en el análisis bivariado simple y se incluyeron posibles variables de interacción. El estar jubilado fue la única variable de interacción que perdió significancia al ajustar el modelo. Asimismo, se encontró que, en el modelo final, era estar enfermo el factor explicativo más importante en la utilización de los servicios de salud por la población anciana sin distinción de género, escolaridad, zona de residencia o estado civil. La derechohabiencia actuó como un factor de confusión debido a que muchos ancianos, con y sin seguridad social, utilizaron los servicios médicos privados o acudieron a otra institución de seguridad social (Cuadro III). 


\section{Discusión}

Los resultados derivados del análisis de la ENSA-II, permiten identificar los factores asociados con la utilización de servicios por la población de 60 años y más y compararlos con los perfiles informados en estudios realizados en otros países. La distribución de las características demográficas y socioeconómicas en dicha encuesta es similar a lo informado en el XI Censo Nacional de Población y Vivienda, excepto que en la ENSA-II se notificó un menor porcentaje de analfabetas. Ambas fuentes coinciden en señalar que el analfabetismo es mayor entre las mujeres, particularmente en una generación cuyas condiciones impedían su acceso a la educación, así como buscar alternativas fuera del hogar.

La distribución por estado civil muestra un patrón que se informa en otros países, ${ }^{23-25}$ donde la mayoría de las mujeres ancianas son casadas, o bien, son viudas debido principalmente a la mayor esperanza de vida. En cuanto a la distribución de la población anciana por género no se encontraron diferencias por zona de residencia (urbana o rural), esto es, las condiciones asociadas con la mayor sobrevivencia de las mujeres respecto de los hombres están presentes tanto en zonas urbanas como en rurales.

Por ocupación se observó que el mayor número de inactivos se encuentra en las zonas urbanas, debido a que la mayoría de los habitantes de zonas rurales no tienen una edad "obligada" para retirarse; al tipo de trabajo que desempeñan en el campo o por que no están en posibilidades de dejar de trabajar por razones económicas. Esto repercute en lo encontrado entre el estado de salud notificado, es decir, el haber trabajado la semana anterior al levantamiento de la encuesta y la zona de residencia, ya que $11 \%$ de los ancianos en zonas rurales que trabajaron la semana anterior al le-

\section{Cuadro III}

ANÁlisis de REgResión logística PARA LA UTILIZACIÓN DE SERVICIOS DE SALUd en la población anciana, ENSA-II. MÉxico, 1994

\begin{tabular}{|c|c|c|c|c|c|c|c|c|}
\hline \multirow[b]{2}{*}{ Variable } & \multicolumn{4}{|c|}{ Completo } & \multicolumn{4}{|c|}{ Final } \\
\hline & OR & Z & $p$ & IC $95 \%$ & $O R$ & Z & $p$ & IC $95 \%$ \\
\hline Enfermedad & 10.31 & 2.17 & 0.030 & $1.25-84.72$ & 17.85 & 25.74 & 0.000 & | $4.33-22.23$ \\
\hline Edad & 0.97 & -2.27 & 0.023 & $0.95-0.99$ & 0.98 & -2.4 & 0.015 & $0.97-0.99$ \\
\hline Trabajó la semana pasada (no) & 2.27 & 3.06 & 0.002 & $1.34-3.83$ & 1.72 & 4.08 & 0.000 & $1.32-2.23$ \\
\hline Derechohabiencia (sí) & 2.34 & 2.34 & 0.000 & $1.56-3.52$ & 2.39 & 7.89 & 0.000 & $1.92-2.97$ \\
\hline Género & 0.98 & 0.98 & 0.929 & $0.625-1.53$ & & & & \\
\hline Sabe leer & 1.53 & 1.53 & 0.076 & $0.96-2.44$ & & & & \\
\hline Zona de residencia (urbana) & 1.17 & 0.45 & 0.650 & $0.69-1.79$ & & & & \\
\hline Estado civil & 1.09 & 0.83 & 0.405 & $0.89-1.32$ & & & & \\
\hline Posprimaria(sí) & 0.89 & -0.38 & 0.707 & $0.52-1.56$ & & & & \\
\hline Jubilado (sí) & 0.67 & -1.38 & 0.167 & $0.38-1.18$ & & & & \\
\hline Enfermedad* Jubilado & 2.31 & 2.12 & 0.034 & $1.06-4.90$ & & & & \\
\hline Enfermedad*Género & 1.29 & 0.91 & 0.363 & $0.74-2.28$ & & & & \\
\hline Enfermedad*Leer & 0.71 & -1.19 & 0.234 & $0.40-1.25$ & & & & \\
\hline Enfermedad*Trabajó & 0.63 & -1.38 & 0.167 & $0.33-1.21$ & & & & \\
\hline Enfermedad* Derecho & 0.92 & -0.32 & 0.744 & $0.55-1.53$ & & & & \\
\hline Enfermedad*Zona & 0.89 & -0.37 & 0.709 & $0.51-1.59$ & & & & \\
\hline Enfermedad*Edo. civil & 0.87 & -1.02 & 0.288 & $0.67-1.24$ & & & & \\
\hline Enfermedad* Edad & 1.01 & 1.07 & 0.286 & $0.99-1.05$ & & & & \\
\hline Enfermedad*Posprimaria & 1.23 & 0.57 & 0.569 & $0.60-2.53$ & & & & \\
\hline
\end{tabular}

Número de observaciones: 4430

Chi cuadrada $=840.39$

Prob. Chi cuadrada $=0.0000$
Número de observaciones: 4588

Chi cuadrada $=870.24$

Prob. Chi cuadrada $=0.0000$

Fuente: Encuesta Nacional de Salud II, Secretaría de Salud, México, 1994 
vantamiento de la encuesta se consideraron en mal estado de salud, a diferencia de $7 \%$ de los que trabajaron en zonas urbanas.

Retirarse de la vida económicamente activa significa un cambio drástico en la vida de una persona; modifica su ritmo de vida; disminuye la actividad física e intelectual así como el ingreso económico; hay menor contacto con amigos o compañeros del trabajo; cuestiones todas que, después de cierto tiempo, pueden conducir a la depresión repercutiendo en la percepción del estado de salud. ${ }^{26,27}$ Así, en la ENSA-II 13.7\% de los ancianos consideraron su salud mala o muy mala, a diferencia de $9 \%$ informado en un estudio realizado entre ancianos asegurados en $\mathrm{EUA}^{28} \mathrm{y}$ de $7 \%$ en personas de 60 años y más en Buenos Aires. Asimismo, se encontró que tanto en Argentina ${ }^{6}$ como en Italia ${ }^{26}$ los hombres tienden a percibir un mejor estado de salud que las mujeres.

Las mujeres utilizan más los servicios médicos, lo cual se podría explicar por los cambios fisiológicos que acompañan su envejecimiento y por la mayor prevalencia de padecimientos como la hipertensión y la diabetes. ${ }^{15}$ Además, debido a cuestiones culturales y de educación, las mujeres se han enfrentado con mayores desigualdades a lo largo de su vida, lo cual, al llegar a la vejez, ejerce un gran efecto sobre su estado de salud; esto es, los problemas como la pérdida de amigos y de apoyo económico, las afecta en mayor grado. Hay un número creciente de viudas que generalmente viven con una pensión muy reducida; las mujeres sufren mayores discapacidades, y, con mayor frecuencia, son víctimas de la violencia. Hay estudios que muestran que las mujeres en edades avanzadas tienden a sufrir más discapacidades y por mayor tiempo que los hombres. ${ }^{12}$

La cobertura de los servicios de salud para la población de 60 años es desigual tanto por lugar de residencia como por grupo de edad y género. Las características de los ancianos en las zonas rurales muestran la inequidad en la prestación de servicios: $82 \%$ carecen de seguridad social y, en el caso de solicitar servicios médicos, primero acuden a los particulares y después a la SSA. Lo anterior se debe a problemas de accesibilidad, disponibilidad y calidad de los servicios de salud en zonas rurales, y ello es comparable con las experiencias de otros países. ${ }^{29}$ Por otra parte, la tasa de hospitalización general (5.5\%) es similar a las de EUA y Costa Rica. ${ }^{26,30}$

Los patrones de consumo de remedios caseros y medicamentos informados en la ENSA-II indican que, independientemente de la severidad del problema de salud, el consumo de medicamentos es muy alto (74\%) y más frecuente en zonas urbanas. En EUA, Finlandia y Argentina las tasas de consumo de medicamentos son similares y varían entre 60 y 90\%. ${ }^{19,31,32}$ En contraste, los remedios caseros son más usados por los habitantes de las zonas rurales, hecho que se puede explicar por la menor disponibilidad de servicios médicos y de medicamentos, así como por los factores culturales relacionados con la curación de las enfermedades.

No se observaron diferencias por género en el consumo de medicamentos; estos resultados concuerdan con lo informado en estudios realizados en otros países. ${ }^{33}$ Sin embargo, en la Encuesta Nacional de Adicciones $^{34}$ se notificó que $24 \%$ de los ancianos de entre 60 y 65 años de edad eran consumidores de drogas médicas, principalmente depresores y opioides, y se encontró un mayor consumo entre las mujeres. Al parecer la tendencia a consumir medicamentos es común; por lo tanto, la sobreprescripción y el exceso de consumo es una consecuencia de la combinación y del número de enfermedades que padecen las personas mayores. El problema de la sobremedicación en los ancianos puede utilizarse como un indicador de la mala atención, del abandono por parte de la familia o de que existe depresión. Un estudio en Suecia informó que casi $25 \%$ de los ingresos hospitalarios están relacionados con efectos adversos a medicamentos y que el riesgo de sufrirlos se incrementa con el número de medicamentos prescritos. ${ }^{31}$

En lo referente a los ancianos que sí utilizaron los servicios de salud en las dos semanas previas al levantamiento de la encuesta, la tasa de utilización $(11.4 \%)$ fue la más alta en el ámbito nacional. Al comparar con resultados de estudios hechos en EUA y Argentina, se observa que al aumentar el periodo de estudio, se incrementan las tasas de utilización, ya que reportan tasas de $59 \%$ en seis meses y de $80 \%$ en un año. ${ }^{6,35}$

En la ENSA-II una proporción considerable (38\%) de los ancianos acude regularmente a los servicios médicos privados. Es difícil comparar esto con la utilización de servicios privados en otros países, no obstante la similitud de las tasas en Italia, Finlandia y Argentina (36, 30 y $29 \%$, respectivamente), ${ }^{6,25,27}$ pues hay que tomar en cuenta la organización y el tipo de financiamiento de los servicios de salud en cada nación. Por ejemplo, en Canadá 100\% de los servicios médicos y hospitalarios son de cobertura universal y financiados por el gobierno federal, por lo que la erogación por el pago de servicios de salud por la población es mínima, mientras que en EUA $96 \%$ de la población anciana está cubierta por Medicare y 77\%, además, paga otro tipo de seguro complementario. ${ }^{36}$ Por el contrario, en México prácticamente todos los servicios privados los paga el paciente salvo cuando se cuenta con un seguro de gastos médicos mayores, que en el caso de los an- 
cianos encuestados en la ENSA-II sólo fue de 1.3\%. Cabe destacar que el costo de los servicios otorgados es percibido por la mayoría de los usuarios como moderado.

La percepción de la calidad del servicio fue considerada como "buena o muy buena" por la mayoría de los ancianos $(80 \%)$, similar a lo notificado en Canadá (77\%) y mucho mayor que lo observado en EUA $(40 \%){ }^{34}$ La satisfacción de los ancianos con los servicios de salud utilizados puede estar influida por factores culturales, por la percepción de la calidad y por la actitud de las personas hacia su propia condición de salud y de vejez. Si una persona anciana cree que envejecer implica un deterioro natural de la salud, acompañado de disminución o fin de la capacidad productiva, pérdida de las funciones físicas y mentales, malestar, dolor e incomodidades, entonces cualquier tipo de atención que se le brinde le parecerá adecuada y de calidad y, en algunos casos, puede considerar, incluso, que no la merece. Esta percepción probablemente no está relacionada con las condiciones reales de salud del anciano, ni con la calidad del servicio proporcionado. Esto puede inferirse al comparar con los grupos de edad más jóvenes, ya que se observa que los ancianos tienden a estar más satisfechos con los servicios de salud.

Las variables que se incluyeron en la ENSA-II permiten acercarse a los criterios del modelo social de Andersen ${ }^{37,38}$ sobre los determinantes de la utilización de los servicios de salud. Este modelo incluye tres grupos de variables: las predisponentes, que abarcan las variables demográficas, de estructura social y las creencias de salud; las facilitadoras como el ingreso económico, el tipo de seguro médico, los recursos de la comunidad, las tasas médico/población, la localización geográfica y la densidad de población, y, por último, las de necesidad como son la percepción del estado de salud por el individuo y la identificación de la necesidad por un profesional de la salud. Tomando como base este modelo, Wolinsky en $1991^{35}$ y Palo Stoller en $1992^{33}$ analizaron la situación de la utilización de servicios médicos por los ancianos en EUA. Wolinsky informó que la percepción del estado de salud es la causa decisiva para la utilización de servicios de salud y no encontró relación con el género, las visitas al médico, el vivir solo y el ser viudo. Por otro lado, la escolaridad sólo tuvo efecto directo sobre el número de visitas al médico; las personas con mayor grado de instrucción visitaron más veces al médico. Por su parte, Stoller encontró que las mujeres consultaron más frecuentemente a los médicos que los hombres y que la escolaridad fue el factor predictor más importante para explicar el contacto con un médico. La edad, la composición del hogar y el número de médicos por habitante también explican un mayor número de visitas al médico, indicando que la utilización de los servicios está definida por la disponibilidad y el acceso. La ENSA-II comparte los hallazgos de estos estudios al encontrar que el estar enfermo es el principal factor que determina la búsqueda de atención médica por los ancianos, a pesar de que las definiciones utilizadas en estos estudios no incluyen el contacto con un médico en las dos semanas previas a la entrevista.

Los resultados señalan que actualmente gran cantidad de ancianos no cuenta con seguridad social, no son económicamente activos y, además, muchos padecen enfermedades crónicas que requieren de vigilancia y atención médica permanente y especializada por parte del personal de salud. En este momento el sistema de salud en México no tiene la capacidad para cubrir esa demanda; de ahí que los ancianos acudan más a los servicios privados, con una repercusión mayor sobre su ingreso y condiciones de vida.

\section{Conclusiones}

Es clara la tendencia de los ancianos a utilizar exclusivamente los servicios curativos, especializados y complejos, debido a las múltiples enfermedades características de esa etapa de la vida. Además, los pocos cuidados preventivos y de mantenimiento de la salud hacen que el costo de la atención de problemas crónicos avanzados para los enfermos y sus familias, así como para las instituciones de salud, sea muy elevado. $7,18,21,25$ Vale la pena destacar que frente a este pronóstico, es imperativo diseñar y poner en efecto una campaña intensiva de prevención para amortiguar la tendencia antes señalada. Existen intervenciones preventivas que, aplicadas oportunamente y dirigidas a grupos de adultos jóvenes, pueden incidir en la prevalencia de afecciones de tipo crónico, tan frecuentes en los ancianos.

Entre las actividades de prevención que mayor impacto y beneficio pueden tener en la población anciana están: la inmunización contra la influenza y neumonía pneumocócica, la prevención de accidentes, así como la detección temprana y el tratamiento oportuno de enfermedades como la tuberculosis, hipertensión, cáncer de mama y cáncer colorrectal. También, detectar deficiencias en los órganos de los sentidos, incontinencia, problemas dentales y podiátricos, niveles de actividad física, estado nutricional y los problemas de funcionalidad. ${ }^{39}$

Ciertamente las necesidades de atención aumentarán en el futuro, y el perfil de morbilidad y mortalidad demandará un tipo de servicio de alto costo, tecnológicamente dependiente y con escaso impacto en la salud de la población general. La infraestructura de servicios para satisfacer dicha demanda, por otro lado, no está 
estratégicamente localizada y, por el otro, no existe el personal de salud preparado para atender de manera integral la salud del anciano. Se requiere conocer las proyecciones de población y realizar estudios sobre la utilización de servicios, para diseñar modelos de prevención, atención y cuidado de la salud que se puedan aplicar a la población de mayor edad. Asimismo, es necesario promover el desarrollo de más investigaciones para conocer las características de salud, las necesidades de atención, así como el costo-beneficio de las diferentes alternativas de atención, en el hogar, en casas-hogar, en centros de salud y en hospitales, que ayudarán a enfrentar esta problemática de salud cuyas dimensiones no han sido correctamente valoradas. Es útil conocer la experiencia de otros países respecto a la atención a la salud de los ancianos considerando los modelos alternativos que se están utilizando, el desarrollo de programas de servicio, sus características y los problemas que se han presentado en su instrumentación, así como los resultados de las evaluaciones que se han realizado.

No es cuestión sólo de crear más y mejores servicios, sino también de inculcar una cultura de la salud: informar, educar y practicar desde la infancia conductas de salud enfocadas a la prevención y diagnóstico temprano de enfermedades. Al mismo tiempo, es necesario echar a andar programas que ayuden al anciano a tener una mejor calidad de vida, ya que muchos padecen enfermedades complejas y dolorosas que hay que diagnosticar, tratar y curar. Esto implica una gran inversión de recursos encaminados a tratar en primera instancia y a prevenir en un plazo mayor.

La vejez debe disfrutarse con más años de vida y de mejor calidad, con la certeza de que serán años verdaderamente agradables en los que una vida de trabajo constante se vea recompensada con la capacidad para realizar todas aquellas actividades recreativas de las que no se pudo disfrutar en otra época de la vida. El reto radica en desarrollar programas y servicios que ayuden al mayor número de personas a alcanzar la tercera edad en condiciones óptimas para disfrutarla.

\section{Referencias}

I. Organización Panamericana de la Salud. Salud de los adultos en las Américas. Documento elaborado por el Programa de Salud del Adulto. Washington, D.C. : Organización Panamericana de la Salud, 1990. 2. Secretaría de Salud. La transición en salud. Origen, rumbo y destino. México, D. F:: Cuadernos de Salud, Secretaría de Salud, 1994:20-33.
3. Organización Panamericana de la Salud. Las condiciones de salud en las Américas. Salud del adulto y del anciano. Publicación científica núm. 524. Washington, D.C.: Organización Panamericana de la Salud, 1990:23-24.

4. Mersel A, Call R, Mann J. Demographic trends of aging Application to gerodontology. Gerodontology 1987;6:9-15.

5. Instituto Nacional de Estadística, Geografía e Informática. Conteo de población y vivienda. México, D. F.: INEGI, 1995.

6. Panamerican Health Organization. Health conditions in the Americas 1994. Scientific publication núm. 549. Washington, D.C.: PAHO, 1994:5556.

7. Anual Report of the Board of Trustees of the Federal Old Age and Survivors Insurance and Disability Insurance Trust Funds. Washington, D.C.: Office of the Actuary, Social Security, 1993.

8. Panamerican Health Organization. A profile of the elderly in Argentina. Techinical paper núm. 26. Washington, D.C.: PAHO,WHO, 1998.

9. Leidl R. Health Economic Issues relevant to Contries with aging populations. World Health Stat Q 1992;45:95-108.

10. United Nations, 1991. Demographic Yearbook. United Nations, Department of Economic and Social Development, Statistical Division. 43a. edición. Nueva York: United Nations, 1992: 105.

II. Consejo Asesor en Epidemiología. México. Información prioritaria en salud. México, D.F.: Dirección General de Epidemiología, Secretaría de Salud, 1990.

12. Secretaría de Salud. Estadísticas vitales 1992. México, D.F.:Subsecretaría de Coordinación y Desarrollo, Dirección General de Estadística Informática y Evaluación, Instituto Nacional de Estadística Geografía e Informática, Dirección General de Estadística, 1993:II2.

13. Peers J.Atención de salud en instituciones para las personas ancianas. Hacia el bienestar de los ancianos. Publicación científica núm. 492 Washington, D.C.: Organización Mundial de la Salud, 1985:77-83.

14. Du-Guerny J. Social policies and planning for midlife and older women in Latin America:An international approach. Psychosocial aspects. Midlife and older women in Latin America and the Caribbean. Washington, D.C.: Pan American Health Organization and American Health Association of Retired Persons, 1990:227-241.

15. Bianco M. Morbidity and mortality of midlife women in Argentina. Health aspects. Midlife and older women in Latin America and Caribbean. Washington, D.C.: Pan American Health Organization and American Association of Retired Persons, 1990:169-177.

16. Nusberg C. El mejoramiento de la atención de la salud del anciano: ejemplos de todo el mundo. Hacia el bienestar de los ancianos. Publicación científica núm. 492. Washington, D.C.: Organización Panamericana de la Salud, 1985:160-165.

17. Secretaría de Salud. Encuesta Nacional de Enfermedades Crónicas 1993. 2a. edición. México, D.F.: Dirección General de Epidemiología, Secretaría de Salud, 1995.

18. Steinbach U. Social networks, institutionalization, and mortality among elderly people in the United States.J Gerontol 1992;47:183S-190S.

19. Boekholdt M. Cuando la atención primaria no basta. El desafío de proporcionar a las personas ancianas una atención institucional accesible, económica y de calidad. Hacia el bienestar de los ancianos. Publicación científica núm. 492. Washington, D.C.: Organización Mundial de la Salud, 1985:7I-76.

20. Taket AR. Resource allocation problems and health services for the elderly. World Health Stat Q 1992;45:89-94.

21. Alvarez-Gutiérrez R, Brown M. Encuesta de las necesidades de los ancianos en México. Salud Publica Mex 1983;25:21-75.

22. Secretaría de Salud. Encuesta Nacional de Salud II. México, D.F.: SSA, 1994.

23. Steel K, Markson E, Crescenzi C, Hoffman S, Bissonnette A. An analysis of types and costs of health care services provided to an elderly inner-city population. Med Care 1992;20:1090-1 100. 
24. Kinsella K. Aging trends in Kenia. J Cross Cult Gerontol 1992;7:259268

25. Jylhä M, Aro S. Social ties and survival among the elderly in Tampere, Finland. Int J Epidemiol 1989;18:158-164.

26. Mulley GP. Preparing for late years. Lancet 1995;345: | 409- | 4 |3.

27. MapelliV. Health needs, demands for health services and expenditures accross social groups in Italy: An empirical investigation. Soc Sci Med 1993;36:999-1009.

28. Soghikian K, Midanik L, Polen M, Ransom L.The effect of retirement on health services utilization: The Kaiser permanent retirement study. J Gerontol 1991;46:358S-360S.

29. Kekki P. Use of illnes-related ambulatory physsician services in Finland. Med Care 1982;20:797-808.

30. Moya de Madrigal L. Patterns of medical service use among midlife and older women in Latin America and the Caribbean. Washington, D.C.: Pan American Health Organization and American Association of Retired Persons, 1990:178-190.

31. Cadigan D, Maganizer J, Fedder D. Polymedicine use among community resident older women: How much of a problem? Am J Public Health 1989;79:1537-1540.
32. Jylhä M.Ten-year change in the use of medical drugs among the elderly -A longitudinal study and cohort comparision.J Clin Epidemiol 1994;47:6979.

33. Sitar D, Boëthius G, Bergman U, Mitenko P. Prescribing patterns for elderly community-Dwelling heavy medicinal drug users in Manitoba, Canada and Jämtland, Sweden. J Clin Epidemiol 1995;48:825-83I.

34. Secretaría de Salud. Encuesta Nacional deAdicciones III. Drogas médicas. México, D.F.: Dirección General de Epidemiología, Secretaría de Salud, 1994. 35. Palo Stoller E. Patterns of physician utilization by the elderly: A multivariate analysis. Med Care 1992;20:1080-1089.

36. Barer M, Hertzman C, Miller R, Pascali M. On being old and sick: The burden of health care for the elderly in Canada and the United States. J Public Health Polit Policy Law 1992; 17:763-782.

37. Wolinsky F, Johnson R. The use of health services by older adults. J Gerontol 199|;4:345S-357S

38. Arredondo A, Meléndez V. Modelos explicativos sobre la utilización de servicios de salud: revisión y análisis. Salud Publica Mex 1992;34:36-39.

39. Winograd C. Assessment of geriatric patients: Scientific American Medicine. Estados Unidos: Scientific American, 1994: I-5. 